\title{
INSIDE LI
}

doi:10.1038/labinvest.2011.197
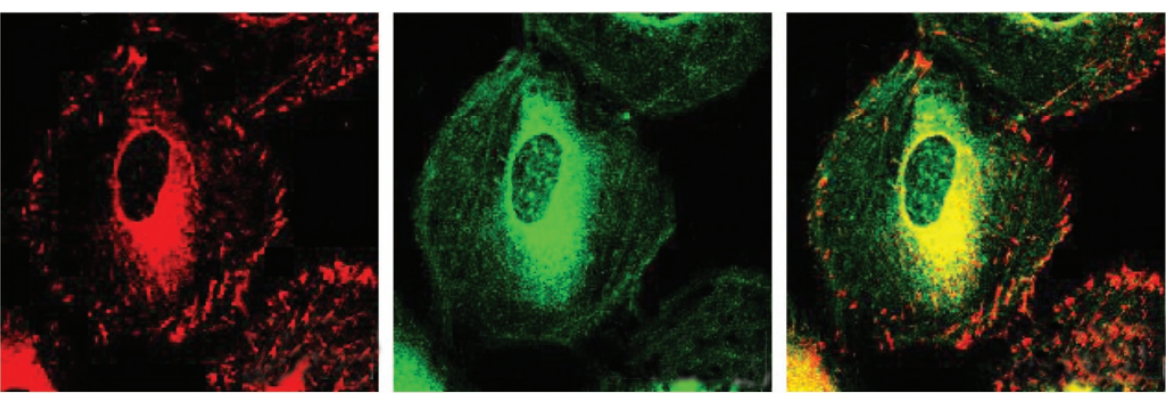

\section{Fascin expression in hepatic stellate cells}

See page 57

Hepatic stellate cells (HSCs) are located in the hepatic sinusoids. When activated by liver injury, HSCs transform into myofibroblast-like cells, which express collagen and contribute to hepatic fibrosis, a major contributor to cirrhosis. It is thought that manipulation of HSCs could be key to preventing end-stage liver disease. Fascin is an actin-bundling protein that has been identified in a variety of both normal cell types and cancer cells. There are three isoforms: fascin-1, -2, and -3. Given its association with the cytoskeleton, fascin is poised to play important roles in cell migration. Uyama and colleagues, knowing that HSCs are rich in actin, postulated that fascin might also play a role in HSCs. They found that fascin was abundantly expressed by HSCs. Interestingly, both activated and quiescent HSCs expressed fascin equally, suggesting that fascin is involved in the function of quiescent as well as stimulated HSCs. Colocalization studies have shown that fascin colocalizes with actin and vinculin, confirming that fascin is involved in the regulation of the cytoskeleton and suggesting that it might interact with focal adhesions. Uyama et al found that depletion of fascin reduced HSC survival in vitro and led to reduced activation of focal adhesion kinase (FAK) and Akt, suggesting that fascin regulates cell survival through FAK-Akt signaling. Furthermore, fascin depletion decreased HSC cell migration in vitro. Taken together, these data identify fascin as a new marker of HSCs and suggest that it plays important roles in maintaining the HSC cytoskeleton and migration. Further studies are required to delineate whether fascin also has integral roles in pathological fibrosis.

\section{Acid disrupts barrier function of squamous epithelium}

See page 22

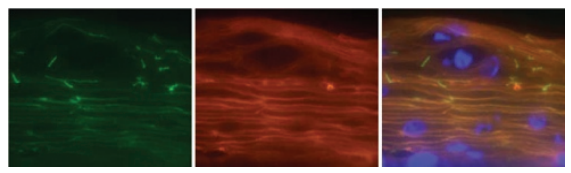

Gastroesophageal reflux disease (GERD) is caused by reflux of acidic stomach contents into the esophagus. Esophageal epithelium plays an important role in preventing damage to the surrounding tissues through its barrier function. However, it has been difficult to study the factors that lead to epithelial damage and disruption of epithelial barrier function. To systematically study these factors, Oshima et al created an in vitro stratified squamous epithelium model. They took advantage of a recent report that rabbit tracheal epithelial cells can be differentiated vitro. Using an air-liquid culture-interface method, they demonstrated that primary human bronchial cells also differentiated into stratified squamous epithelium in vitro, with many similarities to esophageal squamous epithelium. Furthermore, their model showed a tight barrier at culture day 7 . Consistent with barrier function, the model exhibited a distribution of tight junction proteins similar to that observed in normal human esophagus. Interestingly, exposure to acid $(\mathrm{pH} 2)$ disrupted barrier function and resulted in diminished levels of claudin- 4 in the superficial layers. These results indicate into stratified squamous epithelium in that this model recapitulates many of the salient features of esophageal squamous epithelium and should not only provide considerable insight but also potentially serve as a treatment model for GERD and other esophageal diseases.

\section{Epidermal regulation of dermal angiogenesis}

See page 110

Regulation of dermal angiogenesis is critical for normal aspects of skin physiology. Because the epidermis is juxtaposed between the dermis and the external environment, it has been postulated that it might play an important role in regulating dermal angiogenesis. Supporting this hypothesis is the finding that psoriatic epidermis increases dermal angiogenesis via secretion of vasoactive endothelial growth factor. Furthermore, epidermal hypoxia inducible factor 1 (HIF1) appears to play a role in adaptation to environmental oxygen levels. HIF1, a key regulator of hypoxia in normal physiological and pathological responses, is a heterodimeric transcription factor composed of HIF1 $a$ and ARNT (HIF1 $\beta$ )
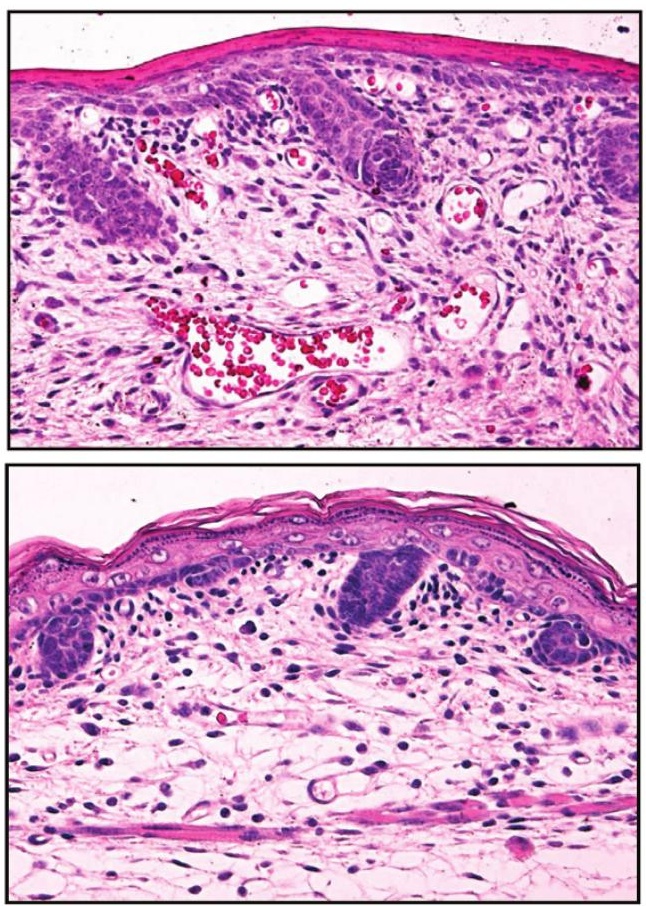
that controls an angiogenic transcription program activated under hypoxic conditions. Because ARNT is ubiquitously expressed, it has been thought that HIF1a is the primary regulator of HIF1 action. However, there is increasing evidence that ARNT levels also regulate HIF1 action. Wondimu et al created an epidermis-specific Arnt mouse knockout to parse Arnt's role in regulating dermal angiogenesis. They found that epidermis-specific Arnt knockout mice had increased levels of dermal capillaries and blood vessels, providing further support that epidermal Hif1 regulates dermal angiogenesis. It appears that loss of Arnt1 is partially compensated by increased expression of Arnt2, which can also dimerize with Hif1a. However, the transcriptional profile of Hif1a/Arnt 1 differs from that of Hif1a/Arnt 2, explaining the increase of dermal angiogenesis. Interestingly, this compensatory mechanism suggests that Hif1 epidermal regulation of dermal angiogenesis is a critical regulatory mechanism. Further studies will be required to elucidate the precise role of epidermal Arnt 1 in normal dermal angiogenesis and abnormal disease states.

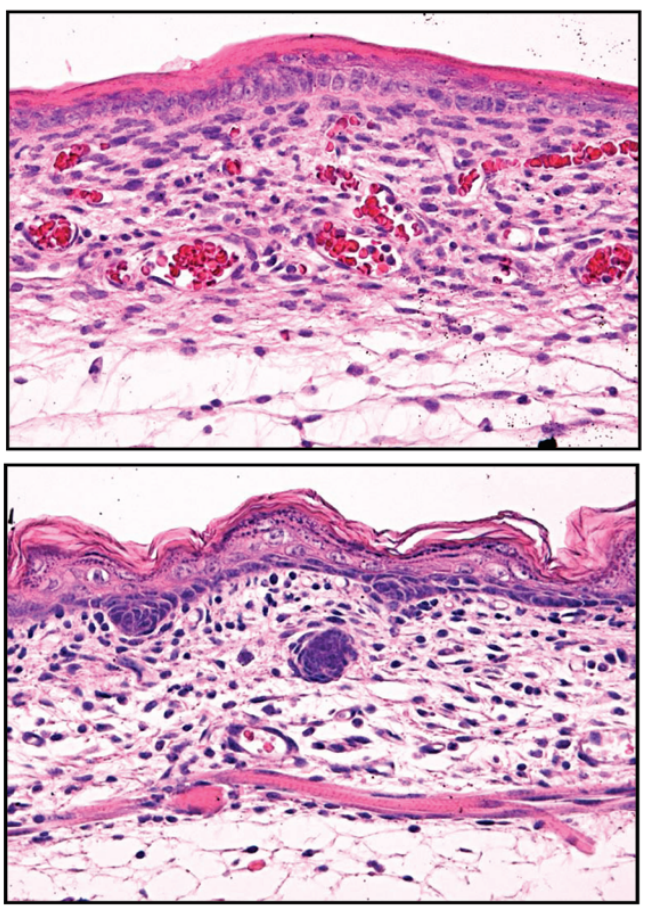

\section{nature.com/pathology}

Breast cancer model resource There is a paucity of in vivo models of breast cancer that authentically recapitulate all the biological features of human breast cancer. To overcome this limitation, DeRose et al, as described in a recent technical report in Nature Medicine, undertook an extensive effort to graft a variety of human

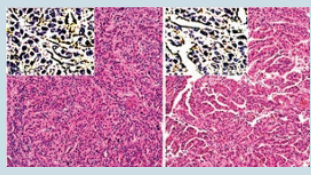
breast cancer specimens directly from patients into cleared mammary fat pads of nonobese diabetic severe combined immunodeficient mice. Only $27 \%$ of the samples took, but those that did exhibited typical human histology and metastasized to clinically relevant sites at high frequency. The investigators also found that tumors that engrafted were associated with a more aggressive clinical course in the patients from whom the grafts were derived, suggesting that engraftment is an inherent feature of more aggressive breast cancers. Nature Medicine 2011;17:1514-1520; doi:10.1038/nm.2454

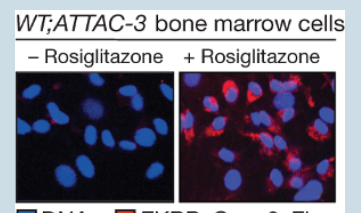

DDNA $\square$ FKBP-Casp8-Flag
Delaying aging-associated disorders Advanced age leads to a wide variety of chronic diseases. However, little is known about the precise mechanisms that underlie age-related maladies. In a recent letter in Nature, Baker et al reported their investigation into whether elimination of senescent cells might delay aging-associated disorders. Using a clever transgenic strategy that allowed them to eliminate $16^{\text {Ink4a }}$-positive senescent cells in a mouse aging model, they found that clearance of senescent cells delayed the onset of age-related pathologies such as loss of muscle and fat. Clearance of senescent cells also reversed muscle and fat deficits in aged mice that had exhibited significant loss of muscle and adipose tissue. These results clearly suggest that strategies to eliminate senescent cells in humans may delay or reverse age-associated disorders.

Nature 2011;479:232-236; doi:10.1038/nature10600

New proteasome inhibitor therapy As recently reported in a letter in Nature Medicine, D'Arcy et al identified a novel compound known as b-AP15 that induces the lysosomal apoptosis pathway. Subsequent analysis revealed that b-AP15 targets the 265 proteasome. Unlike bortezomib - another proteasome inhibitor, which targets the $20 \mathrm{~S}$ proteolytic core particle - it turns out that b-AP15 targets the 195

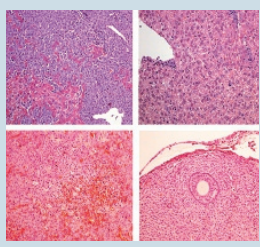
regulatory particle. Specifically, b-AP15 inhibits two 195 regulatory-particle-associated deubiquitinases, ubiquitin C-terminal hydrolase 5 and ubiquitin-specific peptidase 14, leading to the accumulation of polyubiquitinated proteins. Cells treated with b-AP15 have stronger expression of chaperone genes, suggesting that the polyubiquitinated proteins are toxic and thus induce apoptosis. Evaluation of b-AP15 demonstrated that it had remarkable antitumor activity in multiple solid-tumor models and an acute myeloid leukemia model in vivo, suggesting that it may have broad anticancer therapeutic activity in human cancers. Nature Medicine, published online 6 November 2011; doi:10.1038/nm.2536

New insights into the pathogenesis of osteoarthritis Osteoarthritis, which is characterized by the breakdown of articular cartilage in synovial joints, has been thought to be caused by "wear and tear."In a recent letter in Nature Medicine, however, Wang et al present work that challenges this assumption. When

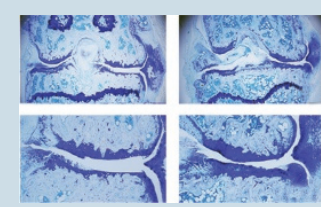
they analyzed the synovial fluid of patients with osteoarthritis using mass spectrometry, the authors found an abundance of complement proteins-specifically, those that compose the membrane attack complex-mediated arm of complement. Gene expression analysis revealed that synovial cells were the source of the complement proteins, which activated chondrocytes to produce degradative enzymes that destroyed the joints. Using genetically engineered mice deficient in components of the complement system, they showed that complement-deficient mice were protected from osteoarthritis in three different osteoarthritis models. These results provide a rationale for targeting the complement system in pursuit of a therapy to prevent or lessen the severity of osteoarthritis.

Nature Medicine, published online 6 November 2011; doi:10.1038/nm.2543 